\title{
Spectroscopic analysis of 31 Aquilae
}

\author{
T.V. Mishenina \\ Astronomical Observatory, Odessa State University, Odessa 270014, Ukraine
}

Received June 12, 1995; accepted March 21, 1996

\begin{abstract}
Aquilae is the only evolved star for which SMR status was confirmed by Taylor (1991). For this reason, a new determination of the atmospheric parameters and chemical composition of 31 Aql has been made. The observational material consists of high-dispersion coudé echelle spectra obtained with a CCD detector and the 1-m telescope of the Special Observatory of the Russian Academy of Sciences. The new value of $[\mathrm{Fe} / \mathrm{H}]$ obtained here is $+0.32 \pm 0.15$ dex. This is close to the upper limit of $[\mathrm{Fe} / \mathrm{H}]$ for dwarf stars belonging to the thin disk of the Galaxy. The value of the $\mathrm{Li}$ abundance $\log A(\mathrm{Li})=1.35$. The value $[C / H]$ obtained here is $-0.05 \pm 0.11$ dex. Within the errors, solar values of [element/Fe] are obtained for $\mathrm{Na}, \mathrm{Y}, \mathrm{Zr}, \mathrm{Ba}, \mathrm{La}, \mathrm{Ce}, \mathrm{Pr}$, and $\mathrm{Nd}$. The age of $31 \mathrm{Aql}$ and its location in the Galaxy are estimated, and a careful analysis of abundance determination errors is carried out. 31 Aql is found to be in the evolutionary stage in which the convective envelope begins to extend.
\end{abstract}

Key words: stars: abundances — stars: evolution — stars: 31 Aql

\section{Introduction}

The notion of super metal rich (SMR) stars was proposed by Spinrad \& Taylor (1969) for stars showing metal abundances far larger than those of the sun. Many researchers have devoted their works to the problem and different hypotheses designed to explain it have been advanced since (in detail, see Gratton \& Sneden 1990; Keenan \& Heck 1994). Later Taylor (1991) made a critical appraisal of published values of the metallicity $[\mathrm{Fe} / \mathrm{H}]$ for 373 evolved $\mathrm{K}$ stars. These stars are of luminosity classes II - IV, and their values of $[\mathrm{Fe} / \mathrm{H}]$ lie between -0.9 and +0.21 dex. Taylor also derived temperature corrections which were applied to a "secondary" data base for 669 stars. In that investigation, the status of super metal rich star was confirmed only for the subgiant 31 Aql. To clarify the status of SMR stars, further work is necessary (Taylor 1991).

It is worth noting that the data for $31 \mathrm{Aql}$ which Taylor considered were limited to results obtained in the 1970s, and that all but one of those results is from photographic spectroscopy. It is also worth noting that Taylor (1996) has published a new statistical investigation of the evidence for SMR stars. Among class IV-V stars, 31 Aql is one of seven stars which Taylor found to be SMR with a confidence level $C>95 \%$.

In this work we study the chemical composition and physical parameters of $31 \mathrm{Aql}$ by using model atmosphere and spectra with a high dispersion and a high signalto-noise ratio. The influence of physical parameters and atomic constants on the abundance determination has been given special consideration.

\section{Observations and reduction}

Spectra of 31 Aql were obtained by Dr. F. Musaev in 1994 September with the 1-m telescope of the Special Astrophysical Observatory of the Russian Academy of Sciences. The coudé echelle spectrometer (Musaev 1993) was used, yielding a resolution $\lambda / \Delta \lambda=36000$. The signal-to-noise ratio of the resulting spectra is larger than 100 per resolution element. The wavelength region $4400-6800 \AA$ was observed, but (except for two Ce II lines) only lines between 5300 and $6700 \AA$ have been used. This wavelength region was chosen because line blending is less than it is at short wavelengths and more reliable continuum placement is possible. A Th-Ar tube was used for the wavelength calibration.

Preliminary reduction of the echelle images was done using the DECH code (Galazutdinov 1992). This program allows one to do flat field division and bias/background subtraction, to extract 1-dimensional spectra from twodimensional images, to take into account diffuse light, to coadd spectra, to remove cosmic-ray hits, and to carry out other necessary procedures. A sample of a reduced spectrum is shown in Fig. 1.

The DECH program also allows one to place a continuum and to measure equivalent widths (EWs). The correctness of the adopted continuum placement is shown by the fact that there is no relation between $\mathrm{Fe}$ 
Table 1. Basic characteristics of $31 \mathrm{Aql}$

\begin{tabular}{crcccccc} 
HD & $*$ & $V$ & $B-V$ & $\pi$ & $\mathrm{Sp}$ & $\log R / R_{\odot}$ & $\log L / L_{\odot}$ \\
\hline 182572 & $31 \mathrm{Aql}$ & 5.16 & 0.77 & 0.057 & $\mathrm{G} 7-\mathrm{IV} \mathrm{H}_{\delta}$ & 0.23 & 0.37 \\
\hline
\end{tabular}

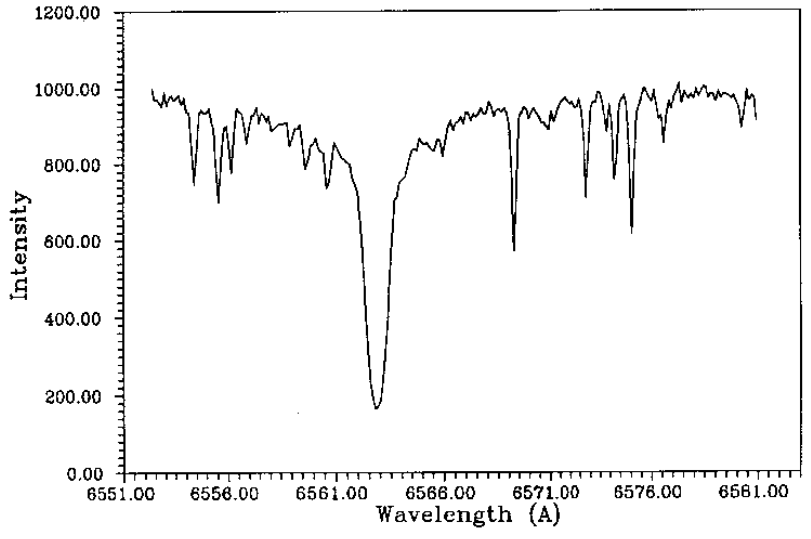

Fig. 1. Fragment of the spectrum of $31 \mathrm{Aql}$ in the region of $\mathrm{H} \alpha$

abundance (from Fe I lines) and wavelength. The EW system

was checked by comparing EWs for $\beta$ Gem with those of Ruland et al. (1980). The mean difference between these two independent sets of EWs, in the sense $\left(\mathrm{EW}_{\text {our }}-\right.$ $\left.\mathrm{EW}_{\mathrm{Rul}}\right)$, is $-0.65 \pm 0.12 \mathrm{~m} \AA(\sigma=0.89 \mathrm{~m} \AA)$. This result is from 57 iron lines.

Basic data for $31 \mathrm{Aql}$ are given in Table 1. The values of the magnitude $V$, the color index $B-V$, the parallax $\pi$, the radius $R / R_{\odot}$, and the absolute luminosity $L / L_{\odot}$ were taken from Malagnini \& Morossi 1990. The spectral type is from Keenan \& Yorka 1988. The space motion $\left(V_{U}, V_{V}\right.$, $\left.V_{W}\right)$ is $\left(+130,-17,-27 \mathrm{~km} \mathrm{~s}^{-1}\right)$ (Eggen 1966).

Table 2. Parameters of atmosphere of $31 \mathrm{Aql}$

\begin{tabular}{ccccl} 
HD & $T_{\text {eff }}$ & $\log g$ & $V_{\mathrm{t}}$ & Ref \\
\hline 182572 & 5600 & 4.20 & 1.2 & this work \\
& 5480 & - & & Rego 1970 \\
& 6000 & - & & Hearnshaw 1971 \\
& 5660 & 4.24 & & Hearnshaw 1972 \\
& 5790 & 4.00 & 0.93 & Glebocki 1972 \\
& 5730 & 4.13 & & Hearnshaw 1975 \\
& 5730 & 4.60 & & Hearnshaw 1976 \\
& 5660 & 4.00 & - & Kuroczkin \& Wisniewski 1977 \\
& 5510 & 3.98 & - & Malagnini \& Morossi 1990 \\
& 5750 & 4.20 & 1.3 & Morell et al. 1992 \\
& 5300 & 3.98 & 1.7 & Berdyugina 1994 \\
& 5708 & - & - & Taylor 1996 \\
\hline
\end{tabular}

\section{Parameter selection for program stars}

Before deriving input atmospheric parameters for $31 \mathrm{Aql}$, it is useful to look at previous results. These are summarized in Table 2.

As one can see from this table, there are differences between the adopted values of $T_{\text {eff }}, \log g$ and $V_{\mathrm{t}}$, but only for $\log g$ are the differences within determination errors. For

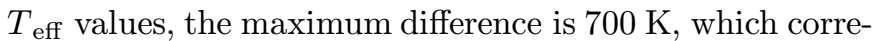
sponds to 0.5 dex in $[\mathrm{Fe} / \mathrm{H}]$. These differences in adopted temperatures are due, first of all, to use of a number of different photometric temperature calibrations. For example, Glebocki (1972) used Johnson's multicolour photometry and effective temperatures based on Carbon \& Gingerich (1969) atmosphere models, and Berdyugina (1994) used McWilliam's (1990) calibration. Thus, the choice of a $T_{\text {eff }}$ calibration is important for abundance determination, and, therefore, for establishing SMR status.

Instead of using temperature calibrations, we have derived the effective temperature of 31 Aql by comparing observed and theoretical $\mathrm{H}_{\alpha}$ line profiles (Kurucz 1979) to those of the sun (Kurucz 1984). The resulting value of $T_{\text {eff }}$ was checked by noting a lack of correlation between excitation potential $\chi$ and abundance from Fe I lines. This test (see Fig. 2) yields $T_{\text {eff }}=5580 \mathrm{~K}$, with a coefficient of correlation of -0.05 for the plotted points. The results of these $T_{\text {eff }}$ determinations are given in Table 3 .

A value of $5600 \mathrm{~K}$ has been adopted for $31 \mathrm{Aql}$. This result is $65 \mathrm{~K}$ lower than the mean value of other authors' determinations.

The surface gravity of $31 \mathrm{Aql}$ was derived from its bolometric magnitude using the standard formula log $g=4$ $\log T_{\text {eff }}+0.4 M_{\text {bol }}+\log \left(M / M_{\odot}\right)-12.5$, where the solar parameters $T_{\text {eff }}=5770 \mathrm{~K}$ and $\log g=4.40$ have been adopted. The absolute magnitude $M_{v}$ was determined using the formula $M_{v}=V+5+5 \log P$, where $P$ is the trigonometric parallax taken from Malagnini \& Morossi (1990). The adopted value of the stellar mass is $1.0 M_{\odot}$ (Malagnini \& Morossi 1990). The gravity has also been determined by forcing Fe II to yield the same total iron abundance as Fe I. The resulting values of $\log g$ are given in Table 3. The gravity derived from ionization balance is slightly larger than that derived by the standard formula, but the two results agree within the limits of determination errors.

Microturbulent velocities $V_{\mathrm{t}}$ were determined by forcing the abundances determined from individual FeI lines to be independent of equivalent width (see Fig. 3). The 
precision of the microturbulent velocity determination is $\pm 0.3 \mathrm{~km} \mathrm{~s}^{-1}$. Corresponding estimates for $\Delta T_{\text {eff }}$ and $\Delta$ $\log g$ are $\pm 100 \mathrm{~K}$ and \pm 0.3 dex, respectively.

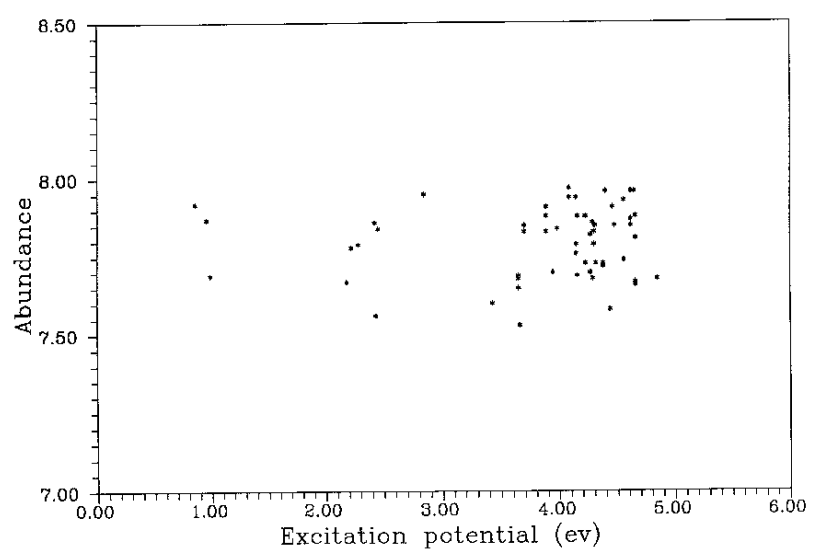

Fig. 2. Dependence between Fe abundance derived from individual $\mathrm{Fe}$ I line and lower excitation potential of this line for $31 \mathrm{Aql}$

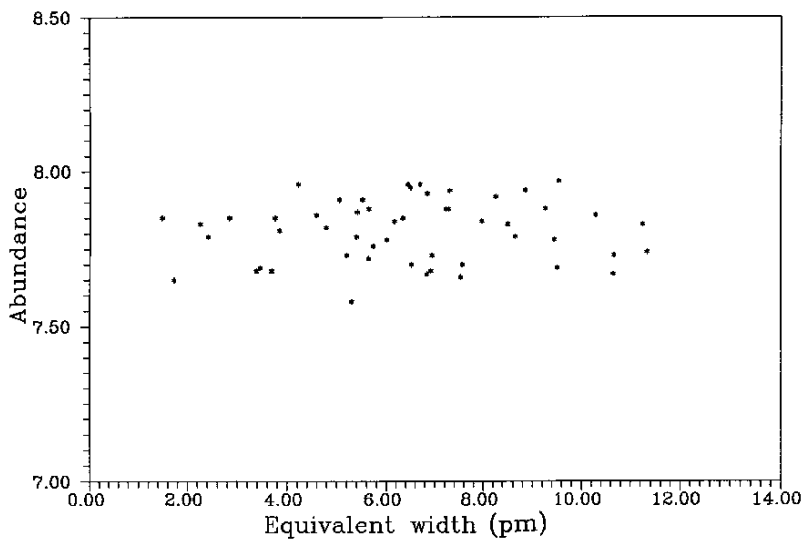

Fig. 3. Dependence between Fe abundance derived from individual Fe I line and its equivalent width

Table 3. Selected atmospheric parameters

\begin{tabular}{rrccc}
$*$ & $T_{\text {eff }}\left(\mathrm{H}_{\alpha}\right)$ & $T_{\text {eff }}(\chi)$ & $\log g\left(M_{v}\right)$ & $\log g(\mathrm{IE})$ \\
\hline $31 \mathrm{Aql}$ & 5640 & 5580 & 4.08 & 4.20 \\
\hline
\end{tabular}

Parameter dependencies for abundance determination are given in Table $4 \mathrm{a}$ (for $[A / H]$ ) and in Table $4 \mathrm{~b}$ (for $[\mathrm{A} / \mathrm{Fe}])$.

\section{Determination of chemical composition}

Synthetic spectra played an important role in the analysis. To calculate such spectra, the new version (STARSP) of the SYNT software (Tsymbal 1980, 1993) was used. This
Table 4. a) Parameter dependencies for abundance determination

\begin{tabular}{lrrrrc}
\multicolumn{1}{c}{ El. } & $\Delta T_{\text {eff }}$ & $\Delta \log g$ & $\Delta[A / H]$ & $\Delta V_{\mathrm{t}}$ & $\Delta$ Tot \\
\hline C I & 0.07 & 0.07 & 0.02 & 0.00 & 0.10 \\
O I & -0.01 & 0.13 & -0.11 & 0.00 & 0.17 \\
Na I & -0.06 & -0.04 & 0.00 & -0.06 & 0.09 \\
Mg I & -0.05 & -0.07 & -0.02 & -0.07 & 0.07 \\
Al I & -0.05 & -0.02 & 0.01 & -0.04 & 0.06 \\
Si I & -0.00 & 0.01 & -0.04 & -0.04 & 0.06 \\
K I & -0.05 & 0.00 & 0.00 & 0.00 & 0.05 \\
Ca I & -0.07 & -0.02 & 0.01 & -0.05 & 0.09 \\
Sc II & 0.01 & 0.11 & -0.10 & -0.05 & 0.16 \\
Ti I & -0.09 & 0.00 & 0.00 & -0.03 & 0.09 \\
V I & -0.11 & 0.01 & 0.00 & -0.03 & 0.11 \\
Cr I & -0.07 & -0.01 & 0.00 & -0.05 & 0.09 \\
Mn I & -0.06 & -0.01 & 0.01 & -0.11 & 0.13 \\
Fe I & -0.06 & -0.02 & -0.02 & -0.09 & 0.11 \\
Fe II & 0.05 & 0.11 & -0.10 & -0.07 & 0.17 \\
Co I & -0.06 & 0.03 & -0.03 & -0.02 & 0.08 \\
Ni I & -0.04 & 0.03 & -0.03 & -0.07 & 0.09 \\
Y II & 0.01 & 0.12 & -0.09 & -0.01 & 0.09 \\
Zr I & -0.12 & 0.01 & -0.01 & 0.00 & 0.12 \\
Ba II & -0.02 & 0.07 & -0.11 & -0.20 & 0.24 \\
La II & -0.02 & 0.13 & -0.11 & -0.01 & 0.17 \\
Ce II & -0.01 & 0.12 & -0.11 & -0.02 & 0.16 \\
Pr II & -0.01 & 0.13 & -0.11 & 0.00 & 0.17 \\
Nd II & -0.01 & 0.13 & -0.12 & -0.01 & 0.18 \\
\hline
\end{tabular}

Notes: $\Delta \log A$ for HD $182572\left(\Delta T_{\text {eff }}=-100 \mathrm{~K}, \Delta \log g=+0.3\right.$ $\left.\operatorname{dex}, \Delta[A / H]=-0.3 \operatorname{dex}, \Delta V_{\mathrm{t}}=+0.3 \mathrm{~km} \mathrm{~s}^{-1}\right)$.

Table 4. b) Parameter dependencies for abundance determination

\begin{tabular}{lrrrrc} 
El. & $\Delta T_{\text {eff }}$ & $\Delta \log g$ & $\Delta[A / H]$ & $\Delta V_{\mathrm{t}}$ & $\Delta$ Tot \\
\hline C I & 0.13 & 0.09 & 0.04 & 0.09 & 0.19 \\
O I & 0.05 & 0.15 & -0.09 & 0.09 & 0.20 \\
Na I & 0.00 & -0.02 & 0.02 & 0.03 & 0.04 \\
Mg I & 0.01 & -0.05 & 0.00 & 0.02 & 0.05 \\
Al I & 0.01 & 0.00 & 0.03 & 0.05 & 0.06 \\
Si I & 0.06 & 0.03 & -0.02 & 0.05 & 0.09 \\
K I & 0.01 & 0.02 & 0.02 & 0.09 & 0.09 \\
Ca I & -0.01 & 0.00 & 0.03 & 0.04 & 0.05 \\
Sc II & 0.07 & 0.13 & -0.08 & 0.04 & 0.17 \\
Ti I & -0.03 & -0.02 & 0.02 & 0.06 & 0.07 \\
V I & -0.05 & 0.03 & 0.02 & 0.06 & 0.09 \\
Cr I & -0.01 & 0.01 & 0.02 & 0.04 & 0.05 \\
Mn I & -0.00 & 0.01 & 0.03 & -0.02 & 0.04 \\
Co I & -0.00 & 0.05 & -0.01 & 0.07 & 0.09 \\
Ni I & 0.02 & 0.05 & -0.01 & 0.02 & 0.06 \\
Y II & 0.07 & 0.14 & -0.07 & 0.08 & 0.19 \\
Zr I & -0.06 & 0.03 & 0.01 & 0.09 & 0.11 \\
Ba II & 0.04 & 0.09 & -0.09 & -0.11 & 0.17 \\
La II & 0.04 & 0.15 & -0.09 & 0.08 & 0.20 \\
Ce II & 0.05 & 0.14 & -0.09 & 0.07 & 0.19 \\
Pr II & 0.05 & 0.15 & -0.09 & 0.09 & 0.20 \\
Nd II & 0.05 & 0.15 & -0.10 & 0.08 & 0.20 \\
\hline
\end{tabular}

Notes: $\Delta \log \mathrm{A} / \mathrm{Fe}$ for HD $182572\left(\Delta T_{\text {eff }}=-100 \mathrm{~K}, \Delta \log g=+0.3\right.$ $\left.\operatorname{dex}, \Delta[A / H]=-0.3 \operatorname{dex}, \Delta V_{\mathrm{t}}=+0.3 \mathrm{~km} \mathrm{~s}^{-1}\right)$. 
code is based on the following assumptions: local thermodynamic equilibrium (LTE), plane-parallel geometry, and hydrostatic equilibrium. The required line lists and the required radiative and collisional damping constants are from Kurucz $(1992,1993)$. The model-atmosphere grid of Kurucz (1992) is used.

The Na abundance of 31 Aql was determined by using synthetic spectra, and such spectra were also used to select lines of $\mathrm{r}$ - and s-process elements which are no more than slightly blended. The original line lists used in the abundance analysis are from Gratton \& Sneden (1990) and Mishenina $(1991,1995)$. Collisional damping constants $\mathrm{C}_{6}$ for Fe I lines specifically were adpoted from Leushin \& Topilskaya (1986; see also Kurucz \& Furenlid 1979). The preferred source of oscillator strengths (log $g f$ values) was Gurtovenko \& Kostyk (1989, designated "GK" below). Oscillator strengths from this source for Sc, V, Mn, Co, and $\mathrm{Eu}$ allow for the effect of hyperfine structure. When log $g f$ values from other sources were used, those sources are cited below.

For elements other than $\mathrm{Na}$ and $\mathrm{Li}$ (see below), the abundance analysis was done by using the WIDTH-9 code of Kurucz. The model atmospheres used in the analysis are from Kurucz (1992). To estimate the influence of this choice on the results, analyses were also done using the model grid of Bell et al. (1976) and its extension to the subgiant region put at our disposal by Bikmaev (1992).

In order to compare results for $31 \mathrm{Aql}$ with solar data, solar abundances were calculated with the $\log g f$ values used here and the solar model of Kurucz (1992). Solar EWs were taken from the work of Gurtovenko \& Kostyk (1989). The lines adopted for the solar analysis specifically are those whose abundances deduced from their EWs are close to the values recommended by Grevesse \& Noels (1993). It may be noted that the solar abundance determination depends on the adopted solar model and solar microturbulent velocity, and that these choices (of course) influence derived relative abundances. For example, the solar $\mathrm{Fe}$ abundances derived from the solar model of Kurucz (1992) are: $\log A(\mathrm{Fe})=7.55$ dex from Fe I lines and $\log A(\mathrm{Fe})=7.50$ dex from Fe II lines. Corresponding results derived from the solar model of Holweger \& Müller (1974) are: $\log A(\mathrm{Fe})=7.63$ dex from Fe I lines and $\log$ $A(\mathrm{Fe})=7.51$ dex from Fe II lines. The difference between these determinations is 0.08 dex for Fe I. A change in the microturbulent velocity by $0.2 \mathrm{~km} \mathrm{~s}^{-1}$ leads to a change in Fe I abundance by 0.03 dex. Solar abundances $\log A_{\odot}$ determined with the Kurucz (1992) solar model (assuming $V_{\mathrm{t}}=1.0 \mathrm{~km} \mathrm{~s}^{-1}$ ) are given in Table 5 .

The final abundance computations carried out with a solar atmosphere model from Kurucz (1992) gave the following results: $T_{\text {eff }}=5600 \mathrm{~K} ; \log g=4.2 ;[A / H]=+0.3$ $\left.\operatorname{dex} ; V_{\mathrm{t}}=1.2 \mathrm{~km} \mathrm{~s}^{-1}\right)$. The results of these computations are given in Table 5 .

\subsection{Li abundances}

The Li abundance was determined by using a modified version of WIDTH-6 (Tsymbal 1994). Allowance was made for absorption by atomic lines and the $\mathrm{CN}$ and $\mathrm{TiO}$ molecules. The required line list is from Orlov \& Shavrina (1984, see also Luck 1977).

\section{2. $C$ and $O$ abundances}

We used the $5380.34 \AA \mathrm{C}$ I and the $6300.31 \AA \quad[\mathrm{O} \mathrm{I}]$ lines to derive $\mathrm{C}$ and $\mathrm{O}$ abundances, respectively. These abundances were determined by means of detailed computations of the molecular dissociation equilibrium. The $\mathrm{C} \mathrm{I}$ line is weak and is isolated in our spectra. The [O I] line is clearly separated from the Sc II line at $6300.69 \AA$. Contributions from an Ni I line at $6300.336 \AA$ and a weak CN line at $6300.265 \AA$ are negligible at the temperature and metallicity of $31 \mathrm{Aql}$, as they are for K giants (Gratton \& Sneden 1990).

The region of the $[\mathrm{O} \mathrm{I}]$ line is contaminated by rather strong telluric features. To remove these features, the spectra of $31 \mathrm{Aql}$ were divided by that of a fast-rotating earlytype star (68 Cyg).

\section{3. $\mathrm{Na}, \mathrm{Mg}, \mathrm{Al}$ abundances}

$\mathrm{Na}$ abundances were derived from the $6154,6160 \AA \mathrm{NaI}$ lines using $\log g f(\mathrm{GK})$ values (see Fig. 4). These NaI lines are practically unblended. The abundance of $\mathrm{Mg}$ was obtained from the $5711.09 \AA \mathrm{Mg}$ I line; the abundance of $\mathrm{Al}$ was obtained from the 6696.02 and $6698.67 \AA \mathrm{Al} \mathrm{I}$ lines.

\subsection{Si, K, Ca, Sc, Ti, V, Cr, Mn, Co, Ni abundances}

The $\mathrm{K}$ abundance was determined from the $5801.75 \AA$ line using a $\log g f$ (solar) value taken from Gratton \& Sneden (1990). The value obtained was compared with a solar abundance from Grevesse \& Noel (1993). The abundances of the other elements noted in the heading of this subsection were determined using the oscillator strengths of Gurtovenko \& Kostyk (1989).

\subsection{Fe abundances}

The final Fe abundance determination was based on 57 Fe I lines and $9 \mathrm{Fe}$ II lines. For the Fe II lines, values of $\log g f(\mathrm{~B}, \mathrm{H})$ are from Biémont et al. (199) and Hannaford et al. (1992). To see if there is a trend of Fe abundance with excitation potential, we have compared the mean $\mathrm{Fe}$ I abundances from lines with high excitation potential $(>3.5 \mathrm{eV}, \sim 50$ lines) and low excitation potential $(\leq 3.5 \mathrm{eV}, \sim 10$ lines). The results obtained show that the dependence of abundance on excitation potential is weak, since the discrepancy is $\sim 0.03$ dex. Since comparatively few low-excitation lines were included in the Fe I line sample, the difference between $\mathrm{Fe}$ abundances derived 
Table 5. Elemental abundances of $31 \mathrm{Aql}$

\begin{tabular}{|c|c|c|c|c|c|c|c|c|}
\hline El. & {$[A / H]$} & $n$ & $\sigma_{1}$ & $\sigma_{2}$ & {$[\mathrm{~A} / \mathrm{Fe}]$} & $\sigma_{3}$ & {$[\mathrm{~A} / \mathrm{Fe}](\mathrm{Ed})$} & $\log A_{\odot}$ \\
\hline & & & \pm & \pm & & \pm & & \\
\hline C I & -0.05 & 1 & - & 0.11 & -0.37 & 0.11 & - & 8.72 \\
\hline O I & 0.25 & 1 & - & 0.11 & -0.07 & 0.11 & - & 8.78 \\
\hline $\mathrm{Na} \mathrm{I}$ & 0.49 & 2 & 0.04 & 0.08 & 0.17 & 0.08 & 0.07 & 6.26 \\
\hline Mg I & 0.28 & 1 & - & 0.11 & -0.04 & 0.11 & 0.11 & 7.59 \\
\hline Al I & 0.49 & 2 & 0.02 & 0.08 & 0.17 & 0.08 & 0.10 & 6.43 \\
\hline Si I & 0.37 & 13 & 0.09 & 0.03 & 0.05 & 0.04 & 0.04 & 7.61 \\
\hline K I & 0.21 & 1 & - & 0.11 & -0.11 & 0.11 & - & 5.21 \\
\hline $\mathrm{Ca} \mathrm{I}$ & 0.36 & 8 & 0.07 & 0.04 & 0.04 & 0.04 & 0.00 & 6.36 \\
\hline Sc II & 0.45 & 9 & 0.12 & 0.04 & 0.13 & 0.04 & - & 2.98 \\
\hline Ti I & 0.42 & 34 & 0.11 & 0.02 & 0.10 & 0.03 & 0.03 & 4.90 \\
\hline V I & 0.46 & 22 & 0.09 & 0.02 & 0.14 & 0.03 & - & 3.84 \\
\hline Cr I & 0.29 & 18 & 0.13 & 0.03 & -0.03 & 0.04 & - & 5.61 \\
\hline Mn I & 0.52 & 3 & 0.04 & 0.07 & 0.20 & 0.07 & - & 5.56 \\
\hline Fe I & 0.32 & 57 & 0.12 & 0.02 & - & - & - & 7.55 \\
\hline $\mathrm{Fe}$ II & 0.29 & 9 & 0.13 & 0.04 & -0.03 & 0.04 & - & 7.50 \\
\hline Co I & 0.40 & 18 & 0.10 & 0.03 & 0.08 & 0.03 & - & 4.83 \\
\hline $\mathrm{Ni} \mathrm{I}$ & 0.33 & 39 & 0.11 & 0.02 & 0.01 & 0.02 & 0.04 & 6.15 \\
\hline Y II & 0.18 & 2 & 0.01 & 0.08 & -0.14 & 0.08 & -0.01 & 2.15 \\
\hline Zr I & 0.27 & 2 & 0.14 & 0.10 & -0.05 & 0.10 & - & 2.36 \\
\hline Ba II & 0.32 & 1 & - & 0.11 & 0.00 & 0.11 & -0.05 & 2.08 \\
\hline La II & 0.35 & 1 & - & 0.11 & 0.03 & 0.11 & - & 0.99 \\
\hline Ce II & 0.25 & 4 & 0.12 & 0.06 & -0.07 & 0.06 & - & 1.47 \\
\hline Pr II & 0.27 & 1 & - & 0.11 & -0.05 & 0.11 & - & 0.67 \\
\hline Nd II & 0.32 & 1 & - & 0.11 & 0.00 & 0.11 & -0.04 & 1.26 \\
\hline
\end{tabular}

Notes: $[A / H]$ - relative abundances for HD $182572, n$ - number of used lines; $\sigma_{1}$ - the rms value of standard deviation per datum; $\sigma_{2}$ - the values from the expression $\max \left[\sigma_{0}, \sigma_{1}\right] / \operatorname{sqrt}(n)$, while " $\sigma_{0}=0.111$ " is an average value of $\sigma_{1}$ from Si I, Ti I, V I, Cr I, Fe I, Ni I, and Co I. For all those elements, $[\mathrm{A} / \mathrm{H}]$ is from at least 13 lines, and $\sigma_{0}$ is about the same for all of them; $[\mathrm{A} / \mathrm{Fe}]-$ relative abundances for HD 182572 ; $\sigma_{3}$ - the same value that $\sigma_{2}$ for $[\mathrm{A} / \mathrm{Fe}] ;[\mathrm{A} / \mathrm{Fe}](\mathrm{Ed})$ - relative abundances for disc dwarfs with $[\mathrm{Fe} / \mathrm{H}]>0.05$ dex from $(\mathrm{Edvardsson}$ et al. 1993); $\log A_{\odot}$ - adopted solar abundances.

from high-excitation lines and from the complete sample is rather small (0.01 dex).

We also made a careful analysis of possible Fe abundance errors due to using different oscillator strength systems and different grids of atmosphere models. The changes from substutituing different oscillator strength systems for determination of relative $\mathrm{Fe}$ abundances changes are lower than 0.03 dex. Specifically, $[\mathrm{Fe} / \mathrm{H}]$ 's for $31 \mathrm{Aql}$ from Fe I and Fe II lines were equal to 0.32 dex and 0.29 dex (Gurtovenko \& Kostyk 1989); 0.32 dex and 0.31 dex (Thévenin et al. 1989; 1990); 0.33 dex and 0.31 dex (Boyarchuk \& Savanov 1985; 1986); and 0.34 dex (Blackwell et al. 1979; 1982) and 0.30 dex (Biémont et al. 1991; Hannaford et al. 1992). In addition, if one substitutes appropriate models from the grid of Bell et al. (1976) for those from the grid of Kurucz (1992), the derived Fe abundance changes by 0.02 dex.

\subsection{Y, Zr, Ba, La, Ce, Pr, Nd abundances}

Abundance determinations for all these atoms except $\mathrm{Ce}$ have been made from 1-2 lines. The Ce abundance was determined from 4 Ce II lines, two from visual region $(\lambda \lambda$ $4523.08,4562.36 \AA)$ and two from the red $(\lambda \lambda$ 5330.58, $5472.30 \AA)$. A discrepancy between Ce abundances deduced from these two line groups was found, and is equal to 0.05 dex.

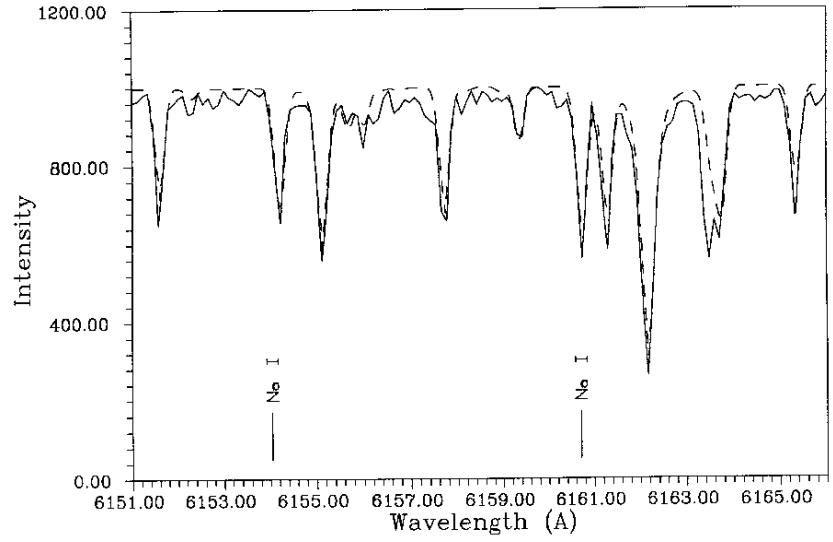

Fig. 4. Comparison between the observed spectrum of $31 \mathrm{Aql}$ (solid line) and synthetic spectrum (dashed line) in the region of $\mathrm{Na}$ I lines $(\log A(\mathrm{Na})=6.75)$

\section{Discussion}

\subsection{The metallicity}

31 Aql was selected because SMR status was confirmed only for this star by Taylor (1991), who considered detailed analyses by previous authors. The results of previous $[\mathrm{Fe} / \mathrm{H}]$ determinations for $31 \mathrm{Aql}$ are given in Table 6 . For the value of $[\mathrm{Fe} / \mathrm{H}]$ given here, the rms error is \pm 0.11 
dex from Fe I lines (see Table 4), but really it is larger. Taking into account errors due to using different oscillator strength systems and grids of atmosphere models, the total error of Fe abundance determination turns to be 0.15 dex (from Fe I lines) and larger than 0.2 dex (from Fe II lines). So, the $[\mathrm{Fe} / \mathrm{H}]$ values obtained in the early works of Hearnshow (1971; 1972) may be larger than ours; the reconsidered $[\mathrm{Fe} / \mathrm{H}]$ values (Hearnshow $1975 ; 1976$ ) and those from papers of Kuroczkin \& Wisniewski (1977) and Morell et al. (1992) agree with ours within the errors of determination.

The $[\mathrm{Fe} / \mathrm{H}]$ value obtained in the present work is close to the upper limit of $[\mathrm{Fe} / \mathrm{H}]$ values for disk dwarfs situated at galactocentric distances of $6-7 \mathrm{kpc}$ (age $\left.-\log \tau_{9}-0.4-1.0\right), 7-8 \mathrm{kpc}\left(\right.$ age $-\log \tau_{9}-0.2-1.0$ ) (Edvardsson et al. 1993). Ages of these dwarfs were estimated by comparing their positions in the H-R diagram with theoretical isochrones. The isochrones were adopted from VandenBerg (1985).

Knowledge of the age of $31 \mathrm{Aql}$ can help one to locate this star in the Galaxy. The age of $31 \mathrm{Aql}$ was derived from thorium by Morell et al. (1992) and it comes to $\log \tau_{9}=$ 0.95 with tracks of VandenBerg (1985). The corresponding galactocentric distance for $31 \mathrm{Aql}$ is probably from 6 to $8 \mathrm{kpc}$.

Table 6. Determinations of metallicities of investigated star

\begin{tabular}{ccll} 
HD & {$[\mathrm{Fe} / \mathrm{H}]$} & Standard & Ref \\
\hline 182572 & 0.32 & Sun & this work \\
& 0.42 & Sun & Rego 1970 \\
& 0.51 & Sun & Hearnshaw 1971 \\
& 0.50 & Sun & Hearnshaw 1972 \\
0.40 & $\epsilon$ Vir & Glebocki 1972 \\
0.28 & Sun & Williams 1972 \\
& 0.44 & Sun & Hearnshaw 1975 \\
0.39 & Sun & Hearnshaw 1976 \\
0.21 & Sun & Kuroczkin \& Wisniewski 1977 \\
& 0.19 & Sun & McWilliam 1990 \\
& 0.30 & Sun & Morell et al. 1992 \\
\hline
\end{tabular}

\section{2. $\mathrm{Li}, \mathrm{C}$ and $\mathrm{O}$ abundances}

Abundances of $\mathrm{Li}$ and $\mathrm{CNO}$ elements are tools for diagnosing processes occurring within stars. When a star advances from the main sequence to the giant branch, the convective envelope begins to extend inward and the surface abundances begin to change. The standard theory predicts that there will be transfer to surface layers of matter enriched by $\mathrm{CN}$-cycle products and depleted in lithium. The $\mathrm{Li}$ abundance obtained here is 1.35 and it agrees with that of Berdyugina (1994) for 31 Aql (taking into account the correction for different $T_{\text {eff }}$ and $V_{\mathrm{t}}$ adopted in these works). This abundance is lower than the primordial $\mathrm{Li}$ value $(\log A(\mathrm{Li})=3.00)$. This result agrees with the present theoretical idea that the convective en- velope grows to contain over half of the mass of the star early in its upward ascent of the giant branch (Iben 1991).

We have found underabundances of $\mathrm{C}$ for 31 Aql. This result likely testifies to the beginning of deep convective mixing in this star. In Fig. 5, an H-R diagram for $31 \mathrm{Aql}$ is plotted, with tracks included for theoretical models of stars of $1.0,1.15$, and $1.3 M_{\odot}$ with overshooting and mass loss (Maeder \& Meynet 1988). The age of 31 Aql deduced from the calculations of Maeder \& Meynet is $\log \tau_{9}=$ 1.00 .

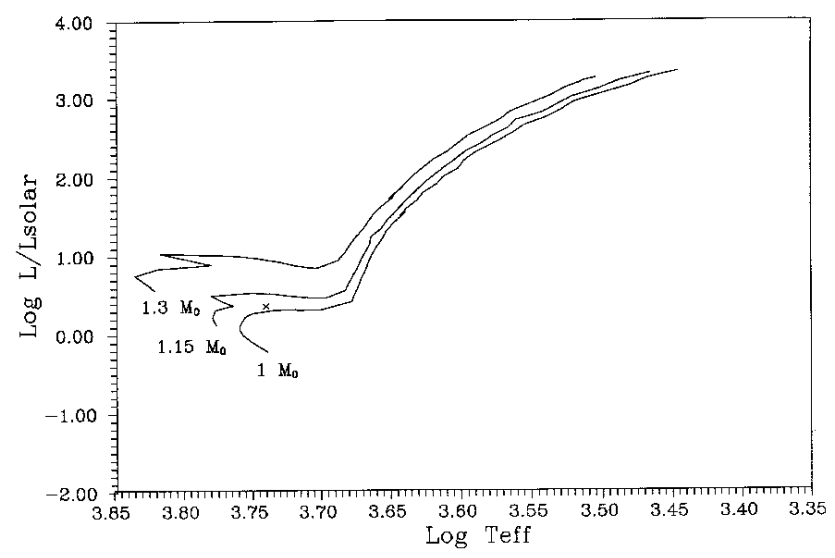

Fig. 5. Tracks in the H-R diagram of theoretical model stars of 1.0, 1.15, and 1.3 M(solar). 31 Aql was marked by cross

\section{3. $\mathrm{Na}, \mathrm{Mg}, \mathrm{Al}$}

We find that $\mathrm{Na}$ and $\mathrm{Al}$ appear to be overabundant in 31 Aql. One possible cause of these results may be non-LTE effects. Departures from LTE in the formation of Na lines in stars with solar metallicity $\left(T_{\text {eff }}=5000-6000 \mathrm{~K}, \log g\right.$ $=3.0-5.0$ ) have been investigated by Mashonkina et al. (1993), who took into account the effects of collisions with $\mathrm{H}$ I atoms. Those authors found that non-LTE corrections for Na I 6154, 6160 lines do not exceed -0.1 dex. For differential investigations of similar stars (such as $31 \mathrm{Aql}$ and the sun), this value is negligible.

$\mathrm{Na}$ (and $\mathrm{Al}$ ) enrichment of the interstellar medium from which 31 Aql was formed may be responsible for the deduced excesses. However, calculations of nucleosynthesis by SNe Ia show that they should make only very small amounts of $\mathrm{Na}$ and $\mathrm{Al}$ (Nomoto et al. 1984). A possible source of $\mathrm{Na}$ enrichment of the interstellar medium might be massive yellow supergiants which explode as Type II SNe (Edvardsson et al. 1993). Edvardsson et al. studied the $\mathrm{NaMgAl}$ star group among the disk dwarfs. The origin of this group is unclear. Moreover, though this group has lower values of $[\mathrm{Fe} / \mathrm{H}]$ than solar, the value of $[\mathrm{Fe} / \mathrm{H}]$ for $31 \mathrm{Aql}$ is larger than solar and its $\mathrm{Al}$ abundance follows its Fe abundance. These results underline the need for further studies of Na synthesis in the Galaxy. 


\subsection{Other elements}

$\mathrm{Si}, \mathrm{Ca}, \mathrm{Ti}$, and Ni abundances relative to iron follow those for thin disk dwarfs at $[\mathrm{Fe} / \mathrm{H}] \sim 0.2 \operatorname{dex}$ (Edvardsson et al. 1993) (see Table 5). The same is true for n-capture elements (Y, Zr, Ba, Nd, La, Ce, Pr), for which the mean value of $[\mathrm{X} / \mathrm{Fe}]$ is $\leq 0$.

\subsection{Comparison with SMR dwarf stars}

As mentioned above, Taylor (1996) has made a new statistical study of SMR-star evidence. Among IV-V class stars, Taylor found a minimum of five which are SMR with a confidence level $C>95 \%$. Two dwarfs from this list have been examined in the extensive investigation of disk dwarfs by Edvardsson et al. (1993): HR 4903 and HR 5325. We have carried out a comparison of the derived abundances for 31 Aql with those for HR 4903 and HR 5325. To diminish the abundance errors due to temperature choice, we have used the abundances relative to iron.

The abundance patterns for $31 \mathrm{Aql}$ and for HR 4903 and HR 5325 are shown in Fig. 6. As is seen in that figure, the pattern of abundances with atomic number is similar for all three stars, with $\mathrm{Mg}$ being an exception (in values of $n$ - capture element abundances is within the errors of determination). One cause of this discrepancy is probably the use of two different wavelength regions to determine $[\mathrm{Mg} / \mathrm{Fe}]$ (the red region for this work, the near-IR for Edvardsson et al. 1993).

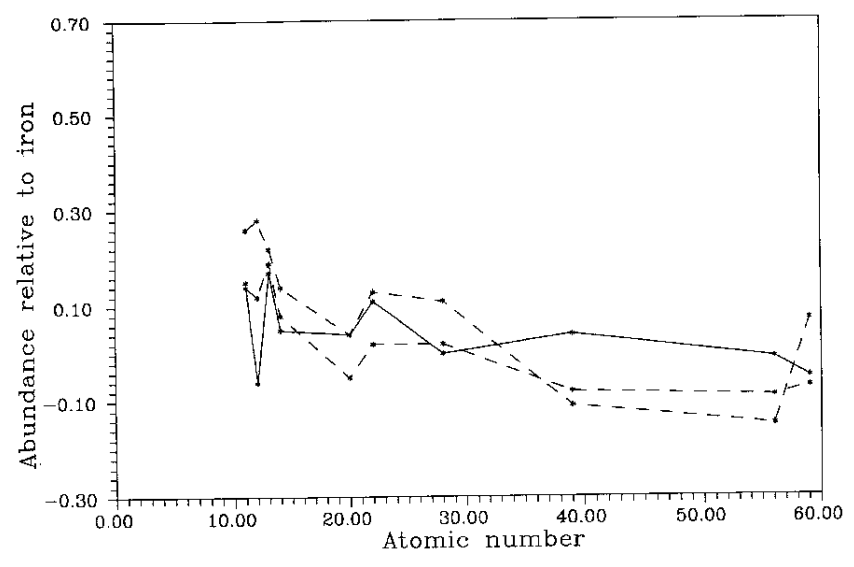

Fig. 6. The abundance patterns for HR 4903 and HR 5325 (dashed, Edvardsson et al. 1993) and for 31 Aql (solid, this work)

\section{Conclusions}

1. The value of $[\mathrm{Fe} / \mathrm{H}]$ obtained for $31 \mathrm{Aql}$ is $+0.32 \pm 0.15$ dex. This result is consistent with the possibility that 31 Aql is an SMR star. The metallicity obtained here is close to upper limit of $[\mathrm{Fe} / \mathrm{H}]$ values for disk dwarfs. $31 \mathrm{Aql}$ is probably a star of thin disk of the Galaxy $(R \sim 6-8 \mathrm{kpc}$, age $\left.-\log \tau_{9} \sim 0.8-1.0\right)$, and is in an evolutionary stage in which the convective envelope begins to extend.

2. The standard method of $V_{\mathrm{t}}$ determination gives a typical subgiant value of $V_{\mathrm{t}}=1.2 \mathrm{~km} \mathrm{~s}^{-1}$.

3. The abundance of $\mathrm{Li}$ is 1.35 . Relative to iron, the $\mathrm{C}$ abundance is lower than the solar value and the $\mathrm{O}$ abundance is slightly lower than the solar value.

4. The $\mathrm{Na}$ and $\mathrm{Al}$ abundances relative to iron have excesses relative to the solar values.

5. Relative to iron, the abundances of $\mathrm{Sr}, \mathrm{Y}, \mathrm{Zr}, \mathrm{Ba}, \mathrm{La}$, $\mathrm{Ce}, \mathrm{Pr}$ and Nd are deficient within the errors of determination.

6. A careful analysis of abundance determination errors shows that the largest contribution to the total error is the error due to the choice of effective temperature.

Acknowledgements. I am grateful to Dr. F. Musaev for the observations, Dr. V. Tsymbal for the modified program, and Dr. B. Taylor for constructive and useful suggestions. I would like also to thank the ASTROPRINT COMPANY for the support during the preparation of this paper.

\section{References}

Bell R.A., Eriksson R., Gustafsson B., Nordlund B., 1976, A\&AS 23, 37

Berdyugina S.V., 1994, Pis'ma v AZh 20, 740

Biémont E., et al., 1991, A\&A 249, 539

Bikmaev I.F., 1992 (private communication)

Blackwell D.E., Petford A.D., Shallis M.J., 1979, MNRAS 186, 657

Blackwell D.E., Petford A.D., Shallis M.J., Simmons G.J., 1982, MNRAS 199, 43

Boyarchuk A., Savanov I.S., 1985, Crimean Astrophys. 70, 57

Boyarchuk A., Savanov I.S., 1986, Crimean Astrophys. 74, 46

Carbon D.E., Gingerich O., 1969, in: Theory and Observations of Normal, Stellar Atmospheres. In: Gingerich O. (ed.). MIT Press, 401

Edvardsson B., Andersen J., Gustafsson B., Lambert D.L., Nissen P.E., Tomkin J., 1993, A\&A 275, 101

Eggen O.J., 1966, Roy. Obs. Bul. 125, E151

Galazutdinov G.A., 1992, Preprint Spets. Astrof. Obs. Russian Glebocki R., 1972, Acta Astron. 22, 141

Gratton R.G., Sneden C., 1990, A\&A 234, 366

Grevesse N., Noels A., 1993, Origin and Evolution of Elements. In: Prantzos N., Vangioni, Flam E., Casse M. (eds.). Cambrige Univ. Press., 14

Gurtovenko E.A., Kostyk R.I., 1989, Fraunhoffer Spectrum and System of Oscillators Strengths, Naukova Dumka, Kiev, p. 200

Hannaford P., et al., 1992, A\&A 259, 301

Hearnshaw J.B., 1971, AJ 168, 109

Hearnshaw J.B., 1972, Mem. Roy. Astr. Soc. 77, 55

Hearnshaw J.B., 1975, A\&A 38, 271

Hearnshaw J.B., 1976, A\&A 51, 85

Holweger H., Müller E.A., 1974, Solar Phys. 39, 19 (HM)

Iben,I. Jr.1991, ApJS 76, 55

Keenan P.C., Yorka S.B., 1988, Bull. Inform. CDS N35, 37 
Keenan P.C., Heck A., 1994, Rev. Mex. Astron. Astrofis. 29, 103

Kuroczkin D., Wieszniewski A., 1977, Acta Astron. 27, 145

Kurucz R.L., 1979, ApJS 40, 1

Kurucz R.L., 1992, Rev. Mex. Astron. Astrofis. 23, 181

Kurucz R.L., 1993 (private communication)

Kurucz R.L., Furenlid I., 1979, SAO Spec. Rep. 387

Kurucz R.L., Furenlid I., Brault J., Testerman L., 1984, Solar Flux, Atlas from 296 to 1300 nm, (Sunspot N.M.: National Solar Observatory)

Leushin V., Topilskaya G., 1986, Astrofizika 25, 103

Luck R.E., 1977, ApJ 218, 752

Maeder A., Meynet G., 1988, A\&AS 76, 411

Malagnini M.L., Morossi C., 1990, A\&AS 85, 1015

Mashonkina L.I., Sachibullin N.A., Shymanski V.V., 1993, AZh 70,372

McWilliam A., 1990, ApJS 74, 1075

Mishenina T.V., Komarov N.S., Kantsen L.E., 1991, Astrofis. Issled. Izv. Spets. Astrofis. Obs. Academii Nauk SSSR 31, 82
Mishenina T.V., Kutsenko S.V., Musaev F.A., 1995, A\&AS 113,333

Morell O., Kallander D., Butcher H.R., 1992, A\&A 259, 543

Musaev F.A., 1993, Pis'ma v AZh 19, 776

Nomoto K., Thielemann F-K., Yokoi K., 1984, ApJ 286, 644

Orlov M.J., Shavrina A.V., 1984, Astrometr. Astrofiz. 51, 15

Rego M.E., 1970, Urania 271, 3

Ruland F., Griffin R., Griffin R., Biehl D. Holweger R., 1980, A\&AS 42, 391

Spinrad H., Taylor B.J., 1969, ApJ 157, 1279

Taylor B.J., 1991, ApJS 76, 715

Taylor B.J., 1996, ApJS 102, 105

Thévenin, et al., 1989, A\&AS 77, 137

Thévenin, et al., 1990, A\&AS 82, 179

Tsymbal V.V., 1980, Synthetic spectra of M-stars, Thesis, Odessa State Univer.

Tsymbal V.V., 1993 (private communication)

Tsymbal V.V., 1994 (private communication)

VandenBerg D.A., 1985, ApJS 58, 711

Williams P.M., 1972, MNRAS 158, 361 